\title{
Multidrug Resistant CTX-M-Producing Escherichia coli: A Growing Threat among HIV Patients in India
}

\author{
Kesavaram Padmavathy, ${ }^{1}$ Krishnan Padma, ${ }^{2}$ and Sikhamani Rajasekaran ${ }^{3}$ \\ ${ }^{1}$ Department of Microbiology, Research Laboratory for Oral and Systemic Health, Sree Balaji Dental College and Hospital, \\ BIHER, Bharath University, Chennai 600100, India \\ ${ }^{2}$ Department of Microbiology, Dr. ALM PGIBMS, University of Madras, Chennai 600113, India \\ ${ }^{3}$ Government Hospital of Thoracic Medicine, Chennai 600047, India
}

Correspondence should be addressed to Krishnan Padma; padma.abpkn@gmail.com

Received 17 December 2015; Accepted 6 March 2016

Academic Editor: Anand S. Deshmukh

Copyright (C) 2016 Kesavaram Padmavathy et al. This is an open access article distributed under the Creative Commons Attribution License, which permits unrestricted use, distribution, and reproduction in any medium, provided the original work is properly cited.

\begin{abstract}
Extended Spectrum $\beta$-Lactamases (ESBLs) confer resistance to third-generation cephalosporins and CTX-M types have emerged as the most prominent ESBLs worldwide. This study was designed to determine the prevalence of CTX-M positive ESBL-producing urinary E. coli isolates from HIV patients and to establish the association of multidrug resistance, phylogeny, and virulence profile with CTX-M production. A total of 57 ESBL producers identified among 76 E. coli strains isolated from HIV patients from South India were screened for $b l a_{\mathrm{CTX}-\mathrm{M}}$, AmpC production, multidrug resistance, and nine virulence associated genes (VAGs), fimH, pap, $a f a / d r a, s f a / f o c$, iutA, fyuA, iroN, usp, and kpsMII. The majority (70.2\%) of the ESBL producers harbored bla $a_{\text {CTX-м }}$ and were AmpC coproducers. Among the CTX-M producers, $47.5 \%$ were found to be UPEC, $10 \%$ harbored as many as 7 VAGs, and $45 \%$ possessed kpsMII. Multidrug resistance $\left(\mathrm{CIP}^{\mathrm{R}} \mathrm{SXT}^{\mathrm{R}} \mathrm{GEN}^{\mathrm{R}}\right)$ was significantly more common among the CTX-M producers compared to the nonproducers (70\% versus $41.2 \%$ ). However, $71.4 \%$ of the multidrug resistant CTX-M producers exhibited susceptibility to nitrofurantoin thereby making it an effective alternative to cephalosporins/fluoroquinolones. The emergence of CTX-M-producing highly virulent, multidrug resistant uropathogenic E. coli is of significant public health concern in countries like India with a high burden of HIV/AIDS.
\end{abstract}

\section{Introduction}

Uropathogenic Escherichia coli (UPEC), a subset of the extraintestinal pathogenic E. coli (ExPEC), is the principal etiological agent of community onset urinary tract infection (UTI) accounting for substantial morbidity and medical costs worldwide. Recent studies have shown that ExPEC isolates have increasingly become resistant to the frontline antibiotics including the cephalosporins, fluoroquinolones, and trimethoprim-sulfamethoxazole [1]. Emergence of $\beta$-lactam resistance mediated by Extended Spectrum $\beta$-Lactamase (ESBLs, e.g., CTX-M types) and AmpC cephalosporinases (especially plasmid mediated AmpC enzymes, e.g., CMY types) is a major global problem [2]. ESBL mediated resistance has been increasingly reported among urinary Escherichia coli isolated from HIV patients in India [3, 4].
ESBL-producing isolates, especially CTX-M-producing E. coli, exhibit an alarming trend in coresistance to other classes of antibiotics [5-7]. Among strains of ExPEC, eleven serogroups $(\mathrm{O} 1, \mathrm{O} 2, \mathrm{O} 4, \mathrm{O} 6, \mathrm{O} 7, \mathrm{O} 8, \mathrm{O} 16, \mathrm{O} 18, \mathrm{O} 25, \mathrm{O} 50$, and O75) are associated with UTI and they generally belong to the virulent phylogroup B2 and to a lesser extent phylogroup $\mathrm{D}$ that elaborate an array of virulence factors including adhesins, protectins, toxins, and iron acquisition systems. Previous studies have reported that multidrug resistant $E$. coli strains are significantly associated with decreased virulence, non-B2 phylogenetic lineage, and host immunocompromised status $[8,9]$. Also, many studies have documented a strong association between CTX-M production and decreased virulence [10-12]. Nevertheless, emergence of ESBL-producing ExPEC with a high virulence potential is of public health concern [13]. There is paucity of information on the virulence 
status and drug resistance profile of CTX-M-producing E. coli particularly infecting the HIV population in our geographical setting. Hence, this study was designed to assess the prevalence of CTX-M producers among the UPEC isolates from HIV patients and to establish the association of phylogeny, multidrug resistance, and virulence profile with CTX-M production.

\section{Materials and Methods}

2.1. Bacterial Isolates. A total of 76 nonrepetitive urinary E. coli isolated from HIV patients (with recent exposure to fluoroquinolones/3G cephalosporins, with CD4 count $<350$ cells $/ \mathrm{mm}^{3}$ and not on antiretroviral therapy, with community onset UTI) from Chennai, South India, were included in the study.

2.2. Detection of ESBL and AmpC Production and Multidrug Resistance. ESBL production was confirmed using cefotaxime (CTX), ceftazidime (CAZ), and cefepime (FEP) alone and in combination with clavulanic acid (CLA) as per clinical and laboratory standards institute (CLSI) guidelines [14]. PCR detection of $b l a_{\mathrm{CTX}-\mathrm{M}}$ was performed among ESBLproducing urinary E. coli isolates from HIV patients [15]. Detection of AmpC phenotype was carried out by boronic acid method [16] and confirmed by AmpC disc test [17]. Antibiotic susceptibility testing was performed as per CLSI to assess the resistance pattern to non-beta-lactam antibiotics [14]. E. coli ATCC 25922 was included as the control. CTX-Mproducing isolates that were resistant to all the three tested antimicrobial classes (fluoroquinolones, ciprofloxacin; folate pathway inhibitor, cotrimoxazole; and aminoglycosides, gentamicin $\left(\mathrm{CIP}^{\mathrm{R}} \mathrm{SXT}^{\mathrm{R}} \mathrm{GEN}^{\mathrm{R}}\right)$ ) were designated as multiple drug resistant isolates.

2.3. Phylogrouping and O Serogrouping. Phylogenetic grouping was done by multiplex PCR (group A $\left(c h u A^{-}\right.$, yja $A^{+/-}$, and $\left.T s p E 4 C 2^{-}\right)$, group $\mathrm{B} 1\left(c h u A^{-}, y j a A^{+/-}\right.$, and $\left.T s p E 4 C 2^{+}\right)$, group $\mathrm{B} 2\left(c h u A^{+}, y j a A^{+}\right.$, and $\left.T s p E 4 C 2^{+/-}\right)$, and group D $\left(c h u A^{+}, y j a A^{-}\right.$, and $\left.\left.T s p E 4 C 2^{+/-}\right)\right)$[18]. O serogrouping was carried out by the traditional antiserum technique at the National Salmonella \& Escherichia Centre, Central Research Institute, Kasauli, Himachal Pradesh, India.

2.4. Virulence Profiling. The isolates were screened for 9 virulence associated genes linked with increased clinical severity: pap [PapA and PapC], sfa/foc [S and F1C fimbriae], afa/dra [Dr-binding afimbrial adhesins] [19], iutA [aerobactin receptor], iroN [salmochelin receptor], fyuA [aerobactin] [20], usp [uropathogenic specific protein] [21], kpsMII [group 2 capsule synthesis] [22], and FimH [type I fimbriae] [23] by PCR. Haemolysin production was assessed on sheep blood agar plates.

2.5. DNA Sequencing. DNA sequencing was performed using Applied Biosystem 3130 Genetic Analyser with ABI PRISM BigDye Terminators version 3.1 (at GenOmb Biotechnologies, Pune, India) to establish positive controls and to confirm the identity of the amplicons. The nucleotide sequences were submitted to the GenBank database under the following accession numbers: bla $a_{\mathrm{CTX}-\mathrm{M}-15}$ (HQ284192), iroN (HQ013325), fyuA (HQ013326), iutA (HM992940), chuA (HQ284193), yjaA (HQ284194), TspE4C2 (HQ284195), papC (HQ165752), sfa (HQ188690), afa (HQ284190), usp (HQ284187), and kpsmII (HQ284189).

2.6. Statistical Methods. Comparisons of proportions between the CTX-M producers and nonproducers were tested using the Chi-square test with Yates correction. Comparison of virulence scores was assessed by Mann-Whitney $U$ test. $p<0.05$ was considered statistically significant.

This study was reviewed and approved by the institutional human ethical committee, Dr. ALM PGIBMS, University of Madras, India.

\section{Results}

Of the 57 ESBL producers screened in this study, 54 (94.7\%) were AmpC producers. The majority $(40 / 57 ; 70.2 \%)$ of the ESBL positive isolates were found to harbor $b l a_{\text {CTX-M. }}$ and were AmpC coproducers.

3.1. Phylogenetic Status and Virulence Profile. The majority of the ESBL producers belonged to the phylogroup D $(27 / 57$, $47.4 \%)$ while phylogroup B2 was the least represented (3/57, $5.3 \%)$. No significant association was observed between $\mathrm{CTX}_{-} \mathrm{M}^{+}$and a specific phylogroup. The distribution of phylogroups among the CTX- $\mathrm{M}^{+}$and $\mathrm{CTX}-\mathrm{M}^{-}$isolates was phylogroup D (20/40, 50\% versus 7/17, 41.2\%), A (9/40, 22.5\% versus $7 / 17,41.2 \%), \mathrm{B} 1$ (9/40, $22.5 \%$ versus $2 / 17,11.8 \%)$, and B2 (2/40, $5 \%$ versus $1 / 17,5.9 \%)$, respectively. Of the 9 VAGs screened, fim $H$ was the most predominant and $s f a / f o c$ was the least detected VAG among both the CTX-M producers and nonproducers (Table 1). None of the CTX-M producers were found to harbor afa. Compared with the CTX-M nonproducers, CTX-M-producing isolates were found to be significantly enriched for $k p s M I I(p=0.048)$, while no statistical difference was observed in the incidence of other VAGs. Maximum virulence score of 7 was exhibited more commonly among the CTX-M producers compared to the nonproducers (10\% versus $5.8 \%$ ). Nonetheless, no statistical difference was observed between the mean virulence score of the CTX-M producers and nonproducers (3.95 versus 3.41, Mann-Whitney $U$ test, $p=0.293)$ (Table 1$)$.

3.2. UPEC. When the UPEC status of the isolates was analyzed based on the operational definition of Johnson et al. [9], that is, presence of $\geq 2$ of the following 5 VAGs, pap, $s f a / f o c, a f a / d r a$, iutA, and kpsMII, it was found that $19 / 40$ (47.5\%) CTX-M-producing isolates qualified as UPEC (mean $=2.53$ ), while $5 / 17$ (29.4\%) of the CTX-M nonproducers were designated as UPEC (mean = 2.4). Of note, there was no statistical difference between the CTX-M producers and nonproducers with regard to the mean scores (Mann-Whitney $U$ test, $p=0.66975)$. Majority of the UPEC isolates of both categories, CTX-M producers $(15 / 19,79 \%)$ and nonproducers $(4 / 5,80 \%)$, belonged to the phylogroup D. 
TABLE 1: VAGs/characteristics among the CTX-M producers $(n=40)$ and nonproducers $(n=17)$.

\begin{tabular}{|c|c|c|c|c|}
\hline \multirow[b]{2}{*}{ VAGs/resistance } & \multicolumn{4}{|c|}{ Number (\%) of strains positive for the VAG/characteristic } \\
\hline & $\begin{array}{l}\text { ESBL producers } \\
\quad(n=57)\end{array}$ & $\begin{array}{c}\text { CTX-M } \\
\text { producers }(n=40)\end{array}$ & $\begin{array}{c}\text { Non-CTX-M } \\
\text { producers }(n=17)\end{array}$ & $p$ value \\
\hline pap & $19(33.3)$ & $16(40)$ & $3(17.7)$ & 0.1833 \\
\hline$s f a / f o c$ & $1(1.8)$ & $0(0)$ & $1(5.9)$ & 0.6564 \\
\hline$a f a / d r a$ & $3(5.3)$ & $2(5)$ & $1(5.9)$ & 0.8914 \\
\hline iutA & $32(56.1)$ & $24(60)$ & $8(47.1)$ & 0.5425 \\
\hline fyuA & $45(78.9)$ & $32(80)$ & $13(76.5)$ & 0.7649 \\
\hline iroN & $20(35.1)$ & $15(37.5)$ & $5(29.4)$ & 0.7779 \\
\hline$k p s M I I$ & $21(36.8)$ & $18(45)$ & $3(17.7)$ & $0.0486^{\mathrm{a}}$ \\
\hline usp & $19(33.3)$ & $11(27.5)$ & $8(47.1)$ & 0.2602 \\
\hline Haemolysis & $3(5.3)$ & $2(5)$ & $1(5.9)$ & 0.8914 \\
\hline$A m p C$ & $54(94.7)$ & $40(100)$ & $14(82.4)$ & $0.0374^{\mathrm{b}}$ \\
\hline $\mathrm{CIP}^{\mathrm{R}}$ & $47(82.5)$ & $37(92.5)$ & $10(58.8)$ & $0.0037^{\mathrm{c}}$ \\
\hline $\mathrm{SXT}^{\mathrm{R}}$ & $57(100)$ & $40(100)$ & $17(100)$ & 1 \\
\hline $\mathrm{GEN}^{\mathrm{R}}$ & $38(66.7)$ & $30(75)$ & $8(47.1)$ & 0.0818 \\
\hline CIP $^{\mathrm{R}} \mathrm{SXT}^{\mathrm{R}} \mathrm{GEN}^{\mathrm{R}}$ & $35(61.4)$ & $28(70)$ & $7(41.2)$ & $0.0403^{\mathrm{d}}$ \\
\hline
\end{tabular}

VAGs: virulence associated genes, $\mathrm{CIP}^{\mathrm{R}}$ : resistant to CIP, $\mathrm{SXT}^{\mathrm{R}}$ : resistant to $\mathrm{SXT}, \mathrm{GEN}^{\mathrm{R}}$ : resistant to GEN, and $\mathrm{CIP}^{\mathrm{R}} \mathrm{SXT}^{\mathrm{R}} \mathrm{GEN}^{\mathrm{R}}$ : multidrug resistance.

${ }^{\text {a }} p<0.05$, odds ratio: 3.8182 , and $95 \%$ CI: 0.9473 to 15.389 .

${ }^{\mathrm{b}} p<0.05$, odds ratio: 32.3665 , and $95 \%$ CI: 2.607 to 401.8328 .

${ }^{\mathrm{c}} p<0.05$, odds ratio: 8.6333 , and $95 \%$ CI: 1.8839 to 39.5634 .

$\mathrm{d}_{p}<0.05$, odds ratio: 3.3333 , and $95 \%$ CI: 1.0252 to 10.8382 .

3.3. Multidrug Resistance. When compared to the CTX-M nonproducers, CTX-M-producing isolates were found to be more resistant to CIP (92.5\% versus 58.8\%) and GEN (75\% versus $47.1 \%)$. All the study isolates were found to exhibit resistance to SXT, but increased susceptibility was observed towards IPM (100\%) and NIT (77.2\%). Further, multidrug resistance $\left(\mathrm{CIP}^{\mathrm{R}} \mathrm{SXT}^{\mathrm{R}} \mathrm{GEN}^{\mathrm{R}}\right)$ was more common among the CTX-M producers compared to the nonproducers (70\% versus $41.2 \%$ ) (Table 1 ). It is noteworthy that $20 / 28$ (71.4\%) of these CTX-M-producing multidrug resistant $\left(\mathrm{CIP}^{\mathrm{R}} \mathrm{SXT}^{\mathrm{R}} \mathrm{GEN}^{\mathrm{R}}\right)$ isolates were susceptible to nitrofurantoin (NIT).

3.4. Serogroups. Among the CTX-M producers, $27.5 \%$ (11/40) of the isolates belonged to the serogroup O25, while 5.9\% (1/17) of the CTX-M nonproducers belonged to the serogroup O25. Also, the majority $(90.9 \%)$ of the $b l a_{\mathrm{CTX}_{-\mathrm{M}^{+}}}$serogroup $\mathrm{O} 25$ isolates belonged to the virulent phylogroups ( $\mathrm{D}(81.8 \%)$ and B2 (9.1\%)), while 9.1\% were of phylogroup A. Of the 11 bla $_{\mathrm{CTX}_{\mathrm{M}} \mathrm{M}^{+}}$serogroup O25 isolates, $100 \%$ were resistant to SXT and CIP and $81.8 \%$ were resistant to GEN; none of the isolates was resistant to imipenem (IPM). Also, 9

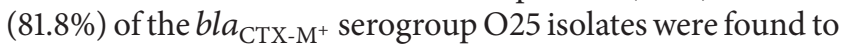
exhibit multidrug resistance $\left(\mathrm{CIP}^{\mathrm{R}} \mathrm{SXT}^{\mathrm{R}} \mathrm{GEN}^{\mathrm{R}}\right)$, while $65.5 \%$ of the $b l a_{\mathrm{CTX}^{-} \mathrm{M}^{+}}$serogroup non-O25 isolates were found to be multidrug resistant. Of the 9 multidrug resistant $b l a_{\text {СТХ-М }}{ }^{+}$ serogroup O25 isolates that were analyzed, 7 (77.8\%) were designated as UPEC with a mean score of 2.9.

\section{Discussion}

The EAU (European Association of Urology) recommends the use of fluoroquinolones, cephalosporins group $3 \mathrm{a} / \mathrm{b}$, for complicated UTI and urosepsis [24]. However, various reports have documented that the emergence of ESBL/AmpC-producing E. coli is a major public health problem and a growing challenge to patient care. Also, ESBLproducing E. coli is designated as a priority drug resistant microbe by the Infectious Diseases Society of America (IDSA) [25]. CTX-M-producing E. coli has emerged worldwide as the leading cause of community onset of UTI in the era of antibiotic resistance [26]. In line with these findings, we report that $70.2 \%$ of the ESBL-producing $E$. coli isolates recovered from HIV patients with UTI were found to harbor $b l a_{\text {CTX-M }}$ which is a matter of concern.

In line with the previous reports, the majority (95\%) of our CTX-M producers belonged to the non-B2 phylogenetic groups, predominantly group D (50\%) [10]. However, another study had reported that CTX-M-producing E. coli causing UTI, predominantly (57.9\%), belong to the low-virulence phylogenetic group B1 [27]. A few recent reports have documented an undoubted link between CTX-M production and reduced virulence [10-12]. Also, previous studies have documented that bacterial strains acquire antibiotic resistance determinants at the expense of their virulence determinants and exhibit a multidrug resistant phenotype [28]. In contrast, our results indicate a high incidence of multidrug resistant, 
virulent E. coli of phylogroup D in our geographical area. It is noteworthy that $47.5 \%$ of the CTX-M producers were found to be UPEC, $10 \%$ had as many as 7 VAGs, and $45 \%$ harbored kpsMII, encoding Group II capsule synthesis. Our results suggest that these CTX-M-producing isolates had a high intrinsic virulence potential. This ascertains the fact that they evade the limited host defences and easily establish an infection in an immunocompromised host. This is in line with another study that reported that CTX-M-producing strains from community outbreaks possess an array of virulence factors that contribute to the fitness and success of these emerging multidrug resistant strains [29]. However, no clonal relationship was observed among our study isolates (data not shown).

Bingen-Bidois et al. had suggested that the concomitant presence of $p a p C$ and $f y u A$ might serve as the minimal requisite for the passage of the ExPEC from the primary focus of infection, the kidney, into the blood stream [30]. A previous study had reported that the presence of plasmid encoded aerobactin was significantly associated with antimicrobial resistance among urosepsis isolates from compromised patients [31]. We propose that ESBL-producing E. coli strains (both the CTX-M producers $(27.5 \%)$ and nonproducers (11.8\%)) carrying pap, fyuA, and iutA constitute a highly uropathogenic genotype that can have a selective advantage for causing urosepsis/bacteremia in these immunocompromised patients.

Currently, fluoroquinolones are widely used in the empirical therapy of UTI. Also, fluoroquinolones are the drug of choice for the treatment of infections caused by ESBLproducing organisms [7]. Nevertheless, a recent study has reported a strong association between ESBL production and fluoroquinolone resistance [32]. In our study, the resistance rate for CIP and GEN in CTX-M producers was remarkably higher than in nonproducers. This is in agreement with other studies that report an increased resistance towards fluoroquinolones, aminoglycosides, and folate pathway inhibitors [7, 33-35]. Though CTX-M-producing isolates showed significant reduction in susceptibility to multiple antibiotics, NIT and IPM retained significant activity.

SXT is routinely being administered to all the HIV subjects as a prophylactic drug for Pneumocystis carinii (jiroveci) pneumonia (PCP) and as a result, we observed that all our study isolates exhibited resistance to SXT. Conversely, none of the study subjects have been exposed to carbapenems which is reflected in the $100 \%$ susceptibility of the E. coli isolates towards IPM.

A short course of NIT has proven to be clinically and microbiologically effective in the treatment of uncomplicated cystitis in women [36]. In line with other reports, we found that majority of our ESBL producers $(77.2 \%)$ were susceptible to NIT (Asha Pai et al. (88.94\%) [37], Garau (71.3\%) [38], Muratani \& Matsumoto (>90\%) [34], and Prakash et al. (73.9\% of CTX-M producers) [33]). An interesting finding is that NIT was found to be effective against the multidrug resistant CTX-M producers (71.4\%) suggesting that NIT may be an effective alternative to cephalosporins and fluoroquinolones.
Multidrug resistance is considered to be a common phenomenon among the CTX-M producers especially towards aminoglycosides, fluoroquinolones, and trimethoprim-sulfamethoxazole $[5,6]$. In line with these reports, a high proportion (70\%) of our CTX-M producers were found to be multidrug resistant $\left(\mathrm{CIP}^{\mathrm{R}} \mathrm{SXT}^{\mathrm{R}} \mathrm{GEN}^{\mathrm{R}}\right)$ suggesting the possibility of treatment failure. Previous antibiotic exposure, especially to cephalosporins and fluoroquinolones, serves as a major risk factor associated with infections caused by ESBL-producing E. coli [39-42]. The selection force exerted by exposure to $\beta$-lactams especially to CTX and CAZ fuels the emergence of divergent, multidrug resistant CTX-M clones. In our study, all the subjects were recently exposed to cephalosporins/fluoroquinolones (for the treatment of lower respiratory infection (LRI) and acute gastroenteritis (AGE), resp.) which might have possibly enhanced the emergence, persistence, and predominance of ESBL- (especially, CTXM-) producing E. coli in the gastrointestinal tract. The intestine serves as the reservoir for the CTX-M producers which in turn colonize and infect the urinary tract in an ascending fashion.

Nearly $28 \%$ of the CTX-M producers belonged to the serogroup O25, of which the majority (81.8\%) were of phylogroup D and one isolate (9.1\%) was of phylogroup B2. Currently, highly virulent, multidrug resistant clone of $E$. coli serotype $\mathrm{O} 25 \mathrm{~b}: \mathrm{H} 4$ belonging to multilocus sequence type 131 (ST131) phylogenetic group B2 producing CTX-M15 has been linked to community onset antimicrobial-resistant infections worldwide including the Indian subcontinent [43-46]. The VAGs commonly described in ST131 E. coli include iha, fimH, sat, kpsMII, fyuA, iutA, usp, traT, ompT, and malX. A recent study had reported that non-ESBL-producing E. coli ST131 isolates were more competitive and virulent than CTX-Mproducing E. coli ST131 isolates. However, bla $a_{\text {CTX-M }}$ positive transconjugants were equally competitive as their susceptible hosts which in turn would favor the global dissemination of CTX-M-producing E. coli ST131 isolates [47]. Though we did not screen specifically for ST131, one fluoroquinolone resistant ESBL/AmpC-producing bla ${ }_{\mathrm{CTX}-\mathrm{M}^{+}}$serotype $\mathrm{O} 25$ isolate that belonged to the phylogroup B2 exhibited multidrug resistance $\left(\mathrm{Cip}^{\mathrm{R}} \mathrm{Co}^{\mathrm{R}} \mathrm{G}^{\mathrm{R}}\right)$ and was multivirulent $\left(p a p^{+}, \mathrm{fimH}^{+}\right.$, $f y u A^{+}, i u t A^{+}, k p s M I I^{+}, u s p^{+}, i r o N^{+}, a f a^{-}$, and $\left.s f a / f o c^{-}\right)$. However, further analysis is needed to confirm whether it belongs to the sequence type 131, a multivirulent clone that has been reported to spread across continents. Of note, this isolate was found to be susceptible to NIT.

\section{Conclusions}

Our results demonstrate a predominance of multidrug resistant CTX-M-producing E. coli of serotype O25, phylogroup $\mathrm{D}$, with multivirulence in our geographical setting (with a high prevalence of HIV infection). We underline the need for continuous surveillance of the emergence and spread of highly virulent CTX-M-producing E. coli in a densely populated country such as India. Dissemination of highly virulent, multidrug resistant CTX-M-producing E. coli is a cause of concern and needs to be considered in the empirical management of UTI among HIV patients. Prudent use of 
antibiotics may serve as an important measure that would reduce antibiotic pressure which in turn could suppress the selection of these multidrug resistant strains.

\section{Competing Interests}

All authors declare that there are no competing interests regarding the publication of this paper.

\section{Acknowledgments}

This work was supported by a financial grant from the Indian Council of Medical Research (ICMR), India. K. Padmavathy was awarded the senior research fellowship by ICMR, India.

\section{References}

[1] B. Foxman, "The epidemiology of urinary tract infection," Nature Reviews Urology, vol. 7, no. 12, pp. 653-660, 2010.

[2] N. Lampri, I. Galani, G. Poulakou et al., "Mecillinam/clavulanate combination: a possible option for the treatment of community-acquired uncomplicated urinary tract infections caused by extended-spectrum $\beta$-lactamase-producing Escherichia coli," Journal of Antimicrobial Chemotherapy, vol. 67, no. 10, pp. 2424-2428, 2012.

[3] R. Vignesh, E. M. Shankar, K. G. Murugavel et al., "Urinary infections due to multi-drug-resistant Escherichia coli among persons with HIV disease at a tertiary AIDS Care Centre in south India," Nephron-Clinical Practice, vol. 110, no. 1, pp. c55c57, 2008.

[4] K. Padmavathy, K. Padma, and S. Rajasekaran, "Extended-spectrum $\beta$-lactamase/AmpC-producing uropathogenic Escherichia coli from HIV patients: do they have a low virulence score?" Journal of Medical Microbiology, vol. 62, no. 3, pp. 345-351, 2013.

[5] S. Nathisuwan, D. S. Burgess, and J. S. Lewis II, "Extended-spectrum $\beta$-lactamases: epidemiology, detection, and treatment," Pharmacotherapy, vol. 21, no. 8, pp. 920-928, 2001.

[6] D. L. Paterson and R. A. Bonomo, "Extended-spectrum $\beta$ lactamases: a clinical update," Clinical Microbiology Reviews, vol. 18, no. 4, pp. 657-686, 2005.

[7] J. D. Pitout and K. B. Laupland, "Extended-spectrum $\beta$ lactamase-producing Enterobacteriaceae: an emerging publichealth concern," The Lancet Infectious Diseases, vol. 8, no. 3, pp. 159-166, 2008.

[8] J. R. Johnson, J. J. Brown, U. B. Carlino, and T. A. Russo, "Colonization with and acquisition of uropathogenic Escherichia coli as revealed by polymerase chain reaction-based detection," Journal of Infectious Diseases, vol. 177, no. 4, pp. 1120-1124, 1998.

[9] J. R. Johnson, M. A. Kuskowski, K. Owens, A. Gajewski, and P. L. Winokur, "Phylogenetic origin and virulence genotype in relation to resistance to fluoroquinolones and/or extendedspectrum cephalosporins and cephamycins among Escherichia coli isolates from animals and humans," Journal of Infectious Diseases, vol. 188, no. 5, pp. 759-768, 2003.

[10] J.-P. Lavigne, H. Marchandin, J. Delmas et al., "qnrA in CTX-Mproducing Escherichia coli isolates from France," Antimicrobial Agents and Chemotherapy, vol. 50, no. 12, pp. 4224-4228, 2006.

[11] C. Branger, O. Zamfir, S. Geoffroy et al., "Genetic background of Escherichia coli and extended-spectrum $\beta$-lactamase type," Emerging Infectious Diseases, vol. 11, no. 1, pp. 54-61, 2005.
[12] V. Leflon-Guibout, C. Jurand, S. Bonacorsi et al., "Emergence and spread, of three clonally related virulent isolates of CTXM-15-producing Escherichia coli with variable resistance to aminoglycosides and tetracycline in a French geriatric hospital," Antimicrobial Agents and Chemotherapy, vol. 48, no. 10, pp. 3736-3742, 2004.

[13] S. Jadhav, A. Hussain, S. Devi et al., "Virulence characteristics and genetic affinities of multiple drug resistant uropathogenic Escherichia coli from a semi urban locality in India," PLOS ONE, vol. 6, no. 3, Article ID e18063, 2011.

[14] Clinical and Laboratory Standards Institute, "Performance standards for antimicrobial susceptibility testing; Twenty third informational supplement," Tech. Rep. M100:S23, 2013.

[15] F.-Y. Chang, L. K. Siu, C.-P. Fung, M.-H. Huang, and M. Ho, "Diversity of SHV and TEM $\beta$-lactamases in Klebsiella pneumoniae: gene evolution in Northern Taiwan and two novel $\beta$-lactamases, SHV-25 and SHV-26," Antimicrobial Agents and Chemotherapy, vol. 45, no. 9, pp. 2407-2413, 2001.

[16] P. E. Coudron, "Inhibitor-based methods for detection of plasmid-mediated AmpC $\beta$-lactamases in Klebsiella spp., Escherichia coli, and Proteus mirabilis," Journal of Clinical Microbiology, vol. 43, no. 8, pp. 4163-4167, 2005.

[17] J. A. Black, E. S. Moland, and K. S. Thomson, "AmpC disk test for detection of plasmid-mediated AmpC $\beta$-lactamases in Enterobacteriaceae lacking chromosomal AmpC $\beta$-lactamases," Journal of Clinical Microbiology, vol. 43, no. 7, pp. 3110-3113, 2005.

[18] O. Clermont, S. Bonacorsi, and E. Bingen, "Rapid and simple determination of the Escherichia coli phylogenetic group," Applied and Environmental Microbiology, vol. 66, no. 10, pp. 4555-4558, 2000.

[19] C. Le Bouguenec, M. Archambaud, and A. Labigne, "Rapid and specific detection of the pap, afa, and sfa adhesin-encoding operons in uropathogenic Escherichia coli strains by polymerase chain reaction," Journal of Clinical Microbiology, vol. 30, no. 5, pp. 1189-1193, 1992.

[20] J. R. Johnson and A. L. Stell, "Extended virulence genotypes of Escherichia coli strains from patients with urosepsis in relation to phylogeny and host compromise," Journal of Infectious Diseases, vol. 181, no. 1, pp. 261-272, 2000.

[21] S. Bekal, R. Brousseau, L. Masson, G. Prefontaine, J. Fairbrother, and J. Harel, "Rapid identification of Escherichia coli pathotypes by virulence gene detection with DNA microarrays," Journal of Clinical Microbiology, vol. 41, no. 5, pp. 2113-2125, 2003.

[22] J. R. Johnson and T. T. O'Bryan, "Detection of the Escherichia coli group 2 polysaccharide capsule synthesis gene $k p s M$ by a rapid and specific PCR-based assay," Journal of Clinical Microbiology, vol. 42, no. 4, pp. 1773-1776, 2004.

[23] S. Y. Tartof, O. D. Solberg, and L. W. Riley, "Genotypic analyses of uropathogenic Escherichia coli based on fimH single nucleotide polymorphisms (SNPs)," Journal of Medical Microbiology, vol. 56, no. 10, pp. 1363-1369, 2007.

[24] M. Grabe, T. E. Bjerklund-Johansen, H. Botto et al., Guidelines on Urological Infections, European Association of Urology, 2013.

[25] G. H. Talbot, J. Bradley, J. E. Edwards Jr., D. Gilbert, M. Scheid, and J. G. Bartlett, "Bad bugs need drugs: an update on the development pipeline from the Antimicrobial Availability Task Force of the Infectious Diseases Society of America," Clinical Infectious Diseases, vol. 42, no. 5, pp. 657-668, 2006.

[26] R. Cantón and T. M. Coque, "The CTX-M $\beta$-lactamase pandemic," Current Opinion in Microbiology, vol. 9, no. 5, pp. 466475, 2006. 
[27] A. L. Paiva, N. Lincopan, K. C. Silva et al., "Low-virulence phylogenetic background of CTX-M-producing Escherichia coli isolated from extraintestinal infections," Journal of Infection in Developing Countries, vol. 7, no. 10, pp. 756-760, 2013.

[28] T. J. Wiles, R. R. Kulesus, and M. A. Mulvey, "Origins and virulence mechanisms of uropathogenic Escherichia coli," Experimental and Molecular Pathology, vol. 85, no. 1, pp. 11-19, 2008.

[29] J. D. D. Pitout, K. B. Laupland, D. L. Church, M. L. Menard, and J. R. Johnson, "Virulence factors of Escherichia coli isolates that produce CTX-M-type extended-spectrum $\beta$-lactamases," Antimicrobial Agents and Chemotherapy, vol. 49, no. 11, pp. 46674670, 2005.

[30] M. Bingen-Bidois, O. Clermont, S. Bonacorsi et al., "Phylogenetic analysis and prevalence of urosepsis strains of Escherichia coli bearing pathogenicity island-like domains," Infection and Immunity, vol. 70, no. 6, pp. 3216-3226, 2002.

[31] J. R. Johnson, S. L. Moseley, P. L. Roberts, and W. E. Stamm, "Aerobactin and other virulence factor genes among strains of Escherichia coli causing urosepsis: association with patient characteristics," Infection and Immunity, vol. 56, no. 2, pp. 405-412, 1988.

[32] T. Frank, J. R. Mbecko, P. Misatou, and D. Monchy, "Emergence of quinolone resistance among extended-spectrum betalactamase-producing Enterobacteriaceae in the Central African Republic: genetic characterization," BMC Research Notes, vol. 4, article 309, 2011.

[33] V. Prakash, J. S. Lewis II, M. L. Herrera, B. L. Wickes, and J. H. Jorgensen, "Oral and parenteral therapeutic options for outpatient urinary infections caused by enterobacteriaceae producing CTX-M extended-spectrum $\beta$-lactamases," Antimicrobial Agents and Chemotherapy, vol. 53, no. 3, pp. 1278-1280, 2009.

[34] T. Muratani and T. Matsumoto, "Urinary tract infection caused by fluoroquinolone- and cephem-resistant Enterobacteriaceae," International Journal of Antimicrobial Agents, vol. 28, supplement 1, pp. S10-S13, 2006.

[35] S. Baby Padmini, B. A. Raju, and K. R. Mani, "Detection of Enterobacteriaceae producing CTX-M extended spectrum $\beta$ lactamases from a tertiary care hospital in south India," Indian Journal of Medical Microbiology, vol. 26, no. 2, pp. 163-166, 2008.

[36] K. Gupta, T. M. Hooton, P. L. Roberts, and W. E. Stamm, "Shortcourse nitrofurantoin for the treatment of acute uncomplicated cystitis in women," Archives of Internal Medicine, vol. 167, no. 20, pp. 2207-2212, 2007.

[37] K. B. Asha Pai, R. Rai, H. Sanjeev, V. K. Karnaker, and M. S. Krishna Prasad, "Nitrofurantoin: an alternative therapy for uncomplicated cystitis in the era of antimicrobial resistance," Journal of Clinical and Diagnostic Research, vol. 5, no. 5, pp. 964966, 2011.

[38] J. Garau, "Other antimicrobials of interest in the era of extended-spectrum $\beta$-lactamases: fosfomycin, nitrofurantoin and tigecycline," Clinical Microbiology and Infection, vol. 14, supplement 1, pp. 198-202, 2008.

[39] J. Rodríguez-Baño and A. Pascual, "Clinical significance of extended-spectrum $\beta$-lactamases," Expert Review of AntiInfective Therapy, vol. 6, no. 5, pp. 671-683, 2008.

[40] D. I. Andersson and D. Hughes, "Antibiotic resistance and its cost: is it possible to reverse resistance?" Nature Reviews Microbiology, vol. 8, no. 4, pp. 260-271, 2010.

[41] R. Cantón and M.-I. Morosini, "Emergence and spread of antibiotic resistance following exposure to antibiotics," FEMS Microbiology Reviews, vol. 35, no. 5, pp. 977-991, 2011.
[42] C. M. Muvunyi, F. Masaisa, C. Bayingana et al., "Decreased susceptibility to commonly used antimicrobial agents in bacterial pathogens isolated from urinary tract infections in Rwanda: need for new antimicrobial guidelines," The American Journal of Tropical Medicine and Hygiene, vol. 84, no. 6, pp. 923-928, 2011.

[43] M.-H. Nicolas-Chanoine, J. Blanco, V. Leflon-Guibout et al., "Intercontinental emergence of Escherichia coli clone O25:H4ST131 producing CTX-M-15," Journal of Antimicrobial Chemotherapy, vol. 61, no. 2, pp. 273-281, 2008.

[44] O. Clermont, M. Lavollay, S. Vimont et al., "The CTX-M-15producing Escherichia coli diffusing clone belongs to a highly virulent B2 phylogenetic subgroup," Journal of Antimicrobial Chemotherapy, vol. 61, no. 5, pp. 1024-1028, 2008.

[45] S. H. Lau, M. E. Kaufmann, D. M. Livermore et al., "UK epidemic Escherichia coli strains A-E, with CTX-M-15 $\beta$-lactamase, all belong to the international O25:H4-ST131 clone," Journal of Antimicrobial Chemotherapy, vol. 62, no. 6, pp. 1241-1244, 2008.

[46] A. Hussain, C. Ewers, N. Nandanwar et al., "Multiresistant uropathogenic Escherichia coli from a region in India where urinary tract infections are endemic: genotypic and phenotypic characteristics of sequence type 131 isolates of the CTX-M-15 extended-spectrum- $\beta$-lactamase-producing lineage," Antimicrobial Agents and Chemotherapy, vol. 56, no. 12, pp. 6358-6365, 2012.

[47] J. Shin and K. S. Ko, "Effect of plasmids harbouring $b l a_{\text {CTX-M }}$ on the virulence and fitness of Escherichia coli ST131 isolates," International Journal of Antimicrobial Agents, vol. 46, no. 2, pp. 214218, 2015. 


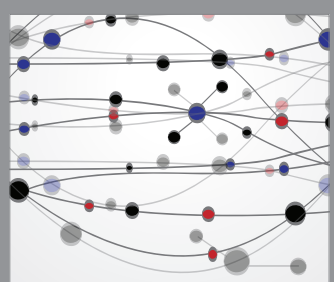

The Scientific World Journal
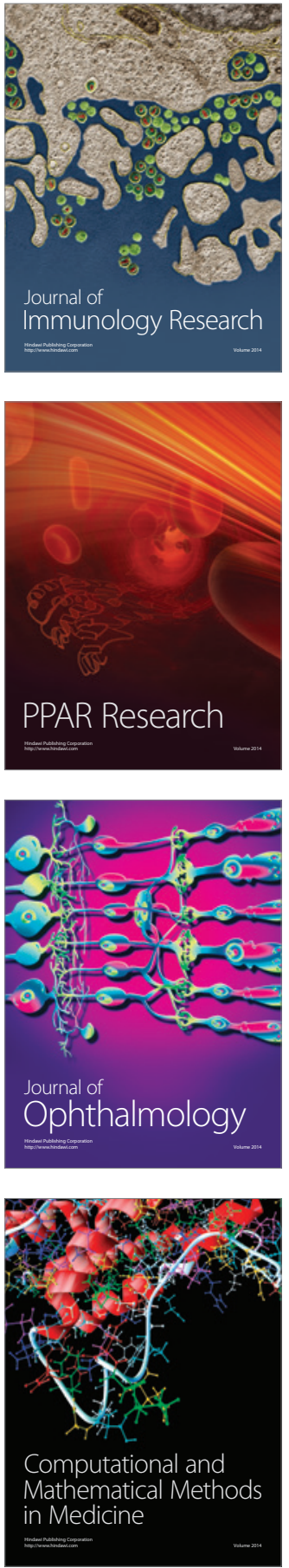

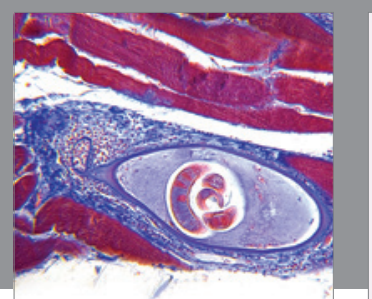

Gastroenterology Research and Practice

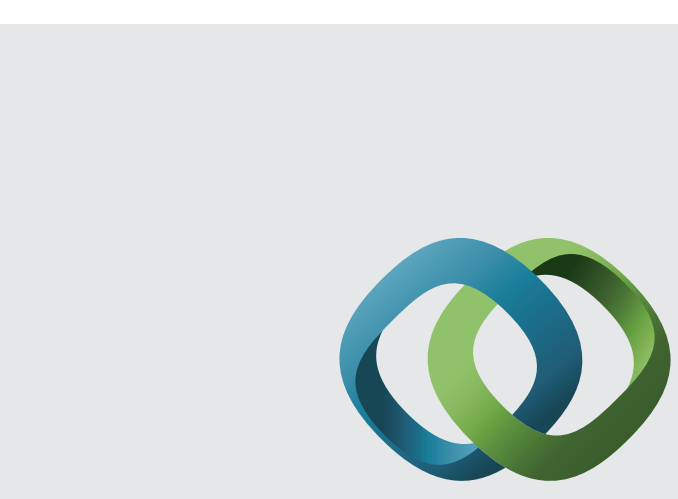

\section{Hindawi}

Submit your manuscripts at

http://www.hindawi.com
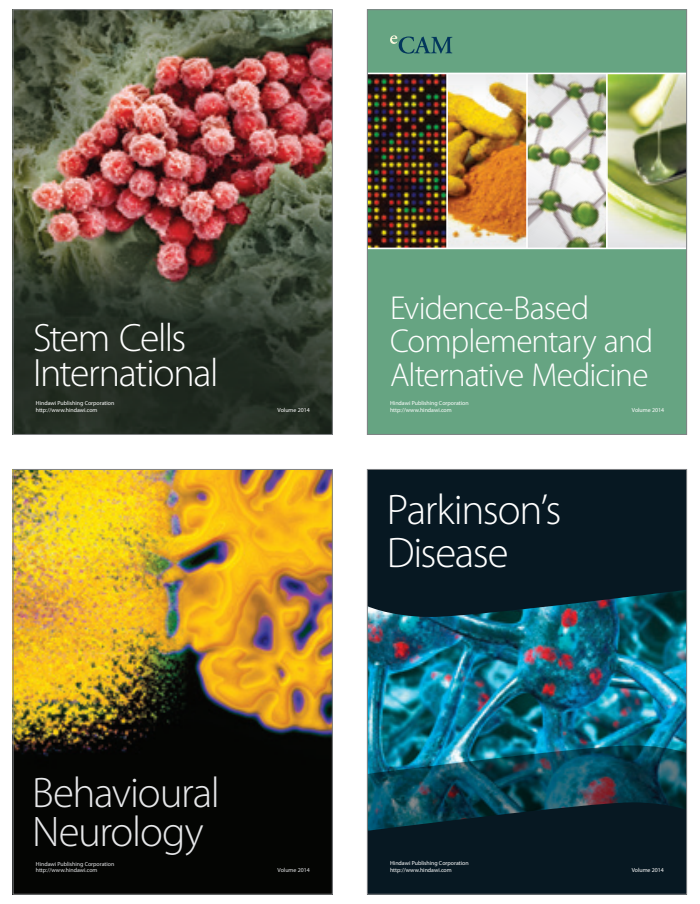
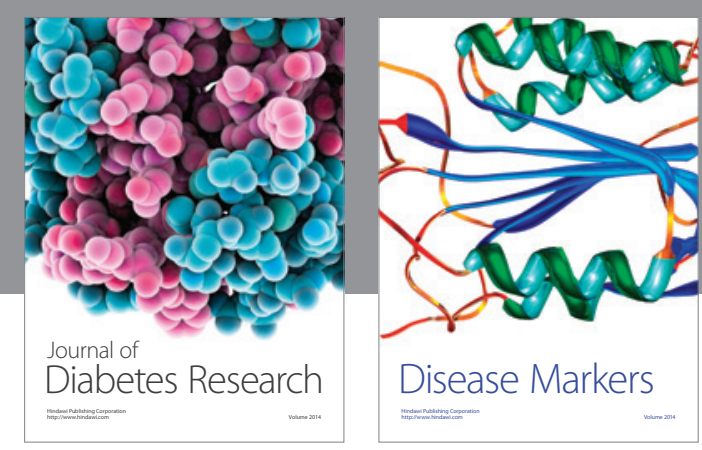

Disease Markers
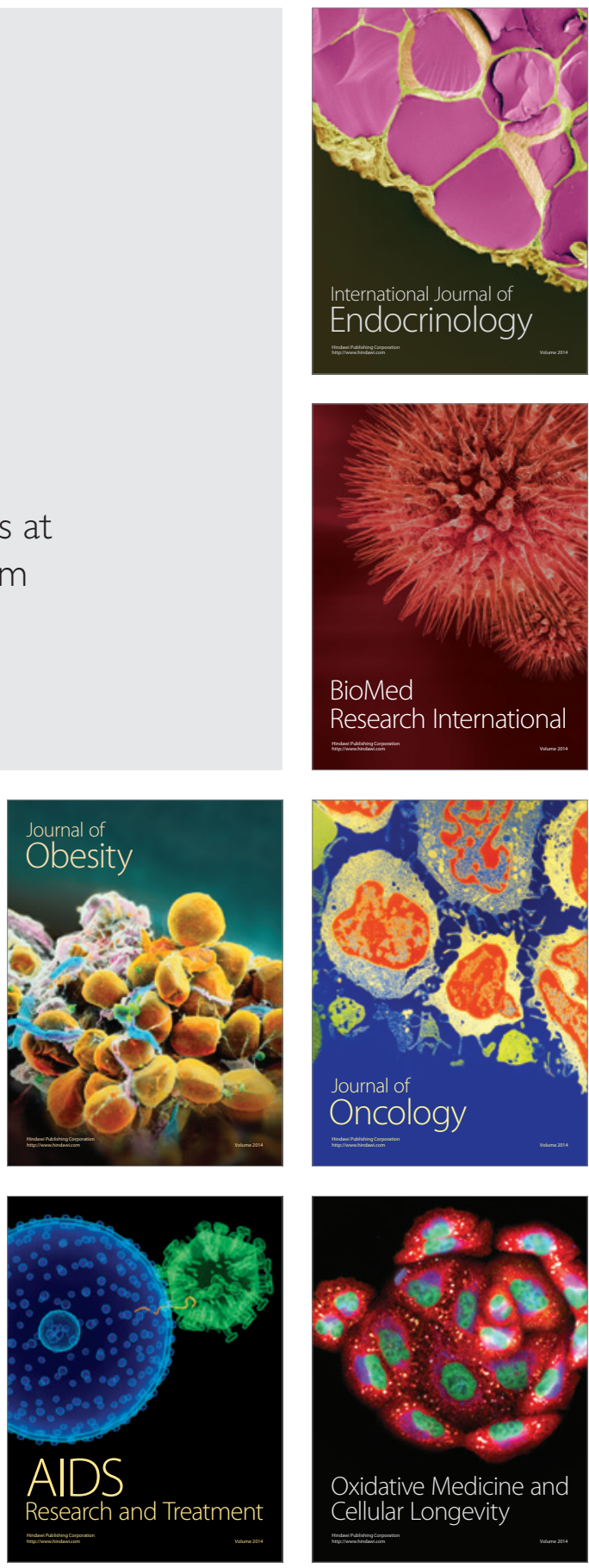